\title{
Is Cholesterol the Key Factor for Autism?
}

\author{
Clifford Lingwood* \\ Molecular Medicine,Hospital for Sick Children, and Depts Laboratory Medicine \& Pathobiology and Biochemistry, University of Toronto,Toronto, Canada
}

*Corresponding author: Clifford Lingwood, Molecular Medicine,Hospital for Sick Children, and Depts Laboratory Medicine \& Pathobiology and Biochemistry, University of Toronto,Toronto, Canada

To Cite This Article: Clifford Lingwood, Is Cholesterol the Key Factor for Autism?. 2020 - 7(6). AJBSR.MS.ID.001206.

DOI: 10.34297/AJBSR.2020.07.001206.

\section{Opinion}

It is hard to reconcile the many genetic and environmental risk factors for ASD [1,2] to a single mechanism. However, modulation of transmembrane signaling by cholesterol and/or glycosphingolipids (GSLs), both enriched in lipid rafts, and their interlinked metabolism [3], could provide the basis of such an all-encompassing axis. Membrane cholesterol is important in neuronal development. $25 \%$ of the body's cholesterol is in myelin. Most GSLs are also in brain, particularly gangliosides, as the name implies. Surprisingly, the neural distribution of cholesterol (and GSLs) remains poorly defined. Abnormal cholesterol metabolism/transport is associated with many neuropathies [4] -Alzheimer's [5], Parkinson's [6], epilepsy [7] and lysosomal storage diseaneuropathies [8,9]. GSL biosynthesis is central in neuronal development [10], particularly myelination [11], and is also aberrant in neurological disease [9,1213].

\section{Cholesterol/GSL complex}

Membrane cholesterol and GSLs accumulate in lipid rafts, ubiquitous eukaryotic signaling foci, central to many pathways, particularly, in this context, neurotransmitter signaling [14]. Rafts are more ordered than non-raft membranes due to the rigid character of both GSLs and cholesterol. GSLs and cholesterol form a complex (1:3) in which we showed the GSL carbohydrate is reoriented from a membrane perpendicular to parallel format. The steroid $\mathrm{OH}$, key for cholesterol binding, is masked by the membrane parallel GSL carbohydrate 'umbrella' $[15,16]$. Membrane parallel GSL is far less available for external ligand binding ('trans' recognition)- but may be more prone to lateral ('cis') ligand binding. The membrane complex serves to reduce trans ligand binding to both GSLs and cholesterol. $\beta$ Methyl cyclodextrin cholesterol extraction remarkably increases tissue and cell GSL 'unmasking' for ligand binding [17]. In cell derived model membranes, cholesterol/GSL masking is also actin dependent [18], suggesting a cytoskeletal role. Cell treatment with a GSL binding ligand e.g. Shigatoxin increased filipin staining of cholesterol, while cell pre-treatment with filipin increased binding of GSL ligands, e.g. Stix, cholera toxin B subunit (binds GM1) [17]. This is a kind of biological 'transistor'.

\section{Common cholesterol ASD pathway?}

Many factors impinge cholesterol levels: transcription [19] (SREBP1/2) [20], microRNA [21], transport [22], translation [23], metabolism [24], degradation [25], ERAD [26], and statins[27]. Cholesterol, in GSL-enriched lipid rafts, could link diverse ASD associated genes. Moreover, the synapse Arc protein, a key to long term memory/synaptic plasticity [28], is related to the HIV retrovirus GAG capsid protein, and transports nascent RNA in virus-like exosome particles in, and between, neuronal cells [29]. HIV virus budding is via the cholesterol dependent [30] exosome pathway [31]. Thus, Arc protein intra/inter neurocellular RNA transport will be cholesterol dependent. In turn, exosome formation requires tetraspanin proteins [32], which bind GSLs [33]. Thus, cholesterol and/ or GSL dependent defects in this essential RNA transport mechanism, could affect a wide range of neurological gene expression, which, in addition to direct cholesterol/GSL roles indicated below, may impinge ASD.

\section{Brain cholesterol is central to ASD}

Several genetic diseases are important in ASD: Smith-Lemli-Opitz Syndrome (SLOS), a cholesterol biosynthesis defect [34], has an $80 \%$ ASD incidence [35]. Low serum cholesterol is associated with ASDs [36]. Moreover, increased dietary cholesterol can ameliorate ASD symptoms [37]. Low cholesterol was found in male ASD individuals [38] and a recent retroactive case-controlled study confirmed the overall hypocholesterolemia of ASD individuals [39], speculating this is a basis for treatment. Furthermore, the cholesterol precursor which accumulates in SLOS, is increased in ASD patients in general and proposed as an autism marker [40]. The mouse model of SLOS shows abnormal development of sero- 
tonin neurons [41], a feature of ASD [42]. SLOS reduces cholesterol controlled [43] sonic hedgehog signaling in vitro [44]. In a significant study [45], the levels of cholesterol in red cells from autistic patients were found consistently lower than normal red cells, and conversely, the level of GM1 GSL, detected using CTB binding, was increased. We contend, based on our GSL masking studies [16] that the lower cholesterol results in decreased masking of GM1 ganglioside in ASD red cells (and all cell types) thereby allowing increased CTB binding. Thus, cholesterol restriction of CTB-GM1 binding is less in these autistic cells. Cerebrospinal fluid GM1[46,47] in autistic children is 'increased' and anti GM1 titre [48] correlates with disease severity. This is consistent with less cholesterol masking of neuronal GM1, and greater membrane fluidity increasing GM1 shedding [49], both promoting GM1 immunogenicity.

In many syndromes in which ASD symptoms are manifest, and animal models of ASD, low cholesterol is a feature [50]. In Sanfilippo Syndrome often misdiagnosed as autism, chondroitin sulfate accumulates [51], including on the cholesterol transporter, apolipoprotein-O [52] to increase regional cholesterol. Fragile X syndrome shows increased ASD incidence [53] and lower cholesterol levels $[54,55]$. Some studies, however, suggest higher ASD cholesterol [56]. Rett syndrome is associated with high cholesterol and ASD [57]. Other genetic syndromes which show ASD-like symptoms and defects in cholesterol metabolism include Cornelia de Lange syndrome [58], tuberous sclerosis complex [59] and Angelman-like syndrome [60]. The serotonin system is abnormal in ASD [61] and the serotonin1A receptor, a key ASD risk factor [62], has a GSL binding site, binds GM1 ganglioside [63] and binds [64] and is modulated by cholesterol [65]. Nerve growth factor (plays a role in ASD verbal defects [66]) receptor is bound/regulated by gangliosides [67]. ASD mTOR signals are abnormal [68] and cholesterol regulates mTOR signaling [69]. Wnt signaling is also a key feature of neurological development and ASD [70] and is regulated by cholesterol [71]. GABA synapse signaling is affected in ASD [72] and the GABA receptor binds cholesterol [73]. PTEN tumor suppressor is associated with ASD [74]. PTEN phosphoinositide dephosphorylation is regulated by cholesterol [75]. Lower brain cholesterol reduces memory/learning [25]. Lower brain cholesterol is a feature of the valproic acid rat model of ASD [76].

The central role of cholesterol in lipid rafts and the central role played by lipid rafts in neurological transmembrane signaling suggests that membrane hypocholesterolemia typical of ASD, could provide the link between the many identified ASD risk factors. The neurological importance of glycosphingolipids and their modulation by/of cholesterol is likely a component of this neurological enigma.

\section{References}

1. Herbert MR (2010) Contributions of the environment and environmentally vulnerable physiology to autism spectrum disorders. Current opinion in neurology 23(2): 103-110.
2. Yuen R. et al (2017) Whole genome sequencing resource identifies 18 new candidate genes for autism spectrum disorder. Nat Neuro sci 20(4): 602-611.

3. Bijl $\mathrm{N}$ et al (2008) The glucosylceramide synthase inhibitor $\mathrm{N}$-(5adamantane-1-yl-methoxy-pentyl)-deoxynojirimycin induces sterol regulatory element-binding protein-regulated gene expression and cholesterol synthesis in HepG2 cells. J Pharmacol Exp Ther 326(3): 849855 .

4. Petrov AM, Kasimov MR \& Zefirov AL (2017) Cholesterol in the Pathogenesis of Alzheimer's, Parkinson's Diseases and Autism: Link to Synaptic Dysfunction. Acta Naturae 9(1): 26-37.

5. Di Scala C, Chahinian H, Yahi N, Garmy N \& Fantini J (2014) Interaction of Alzheimer's beta-amyloid peptides with cholesterol: mechanistic insights into amyloid pore formation. Biochemistry 53(28): 4489-4502

6. Fantini J, Carlus D \& Yahi N (2011) The fusogenic tilted peptide (67-78) of alpha-synuclein is a cholesterol binding domain. Biochim Biophys Acta 1808(10): 2343-2351.

7. Jozwiak S, Kossoff EH, Kotulska \& Jozwiak K (2011) Dietary treatment of epilepsy: rebirth of an ancient treatment. Neurologia i neurochirurgia polska 45(4): 370-378

8. Vanier MT (1999) Lipid changes in Niemann-Pick disease type C brain: personal experience and review of the literature. Neurochem Res 24(4): 481-489.

9. Gondre Lewis MC, McGlynn R \& Walkley SU (2003) Cholesterol accumulation in NPC1-deficient neurons is ganglioside dependent. Current biology: CB 13(15): 1324-1329.

10. Ngamukote S, Yanagisawa M, Ariga T, Ando S \& Yu RK (2007) Developmental changes of glycosphingolipids and expression of glycogenes in mouse brains. Journal of neurochemistry 103(6): 23272341.

11. Stoffel W \& Bosio A (1997) Myelin glycolipids and their functions. Curr Opin Neurobiol 7(5): 654-661.

12. Davidson CD et al (2009) Chronic cyclodextrin treatment of murine Niemann-Pick C disease ameliorates neuronal cholesterol and glycosphingolipid storage and disease progression. PLoS One 4(9): e6951.

13. Hashimoto $\mathrm{N}$ et al (2016) Cholesterol-dependent increases in glucosylceramide synthase activity in Niemann-Pick disease type C model cells: Abnormal trafficking of endogenously formed ceramide metabolites by inhibition of the enzyme. Neuropharmacology 110 (ptA): 458-469.

14. Fantini J \& Barrantes FJ (2009) Sphingolipid/cholesterol regulation of neurotransmitter receptor conformation and function. Biochim Biophys Acta 1788(1): 2345-2361.

15. Yahi N, Aulas A \& Fantini J (2010) How cholesterol constrains glycolipid conformation for optimal recognition of Alzheimer's beta amyloid peptide (Abeta1-40). PLoS One 5(2): e9079.

16. Lingwood D et al (2011) Cholesterol modulates glycolipid conformation and receptor activity. Nature Chem. Biol 7(5): 260-262.

17. Novak A et al (2013) Cholesterol masking membrane glycosphingolipid tumor-associated antigens reduces their immunodetection in human cancer biopsies. Glycobiology 23(11): 1230-1239.

18. Mahfoud R, Manis A, Binnington B, Ackerley C \& Lingwood CA (2010) A major fraction of glycosphingolipids in model and cellular cholesterol containing membranes are undetectable by their binding proteins. J Biol Chem 285(46): 36049-36059.

19. Pacheco NL et al (2017) RNA sequencing and proteomics approaches reveal novel deficits in the cortex of Mecp2-deficient mice, a model for Rett syndrome. Mol Autism 8: 56.

20. Brown MS \& Goldstein JL (1997) The SREBP pathway: regulation of cholesterol metabolism by proteolysis of a membrane-bound transcription factor. Cell 89(3): 331-340. 
21. Jeon TI \& Osborne TF (2016) miRNA and cholesterol homeostasis. Biochim Biophys Acta 1861(12): 2041-2046.

22. Litvinov DY, Savushkin EV \& Dergunov AD (2018) Intracellular and Plasma Membrane Events in Cholesterol Transport and Homeostasis. J Lipids 3965054.

23. Chambers CM \& Ness GC (1998) Dietary cholesterol regulates hepatic 3-hydroxy-3-methylglutaryl coenzyme A reductase gene expression in rats primarily at the level of translation. Arch Biochem Biophys 354(2): 317-322.

24. Suresh S, Yan Z, Patel RC, Patel YC \& Patel SC (1998) Cellular cholesterol storage in the Niemann-Pick disease type C mouse is associated with increased expression and defective processing of apolipoprotein D. Journal of neurochemistry 70(1): 242-251.

25. Mast N, Lin J, Anderson KW, Bjorke I \& Pikuleva IA (2017) Transcriptional and post-translational changes in the brain of mice deficient in cholesterol removal mediated by cytochrome P450 46A1 (CYP46A1). PLoS One 12(10): e0187168.

26. DeBose \& Boyd RA (2008) Feedback regulation of cholesterol synthesis: sterol-accelerated ubiquitination and degradation of HMG CoA reductase. Cell research 18(6): 609-621.

27. Tatley M \& Savage R (2007) Psychiatric adverse reactions with statins, fibrates and ezetimibe: implications for the use of lipid-lowering agents. Drug safety: an international journal of medical toxicology and drug experience 30(3): 195-201.

28. Martinez MC, Alen N, Ballarini F, Moncada D \& Viola H (2012) Memory traces compete under regimes of limited Arc protein synthesis: implications for memory interference. Neurobiol Learn Mem 98(2): 165-173.

29. Pastuzyn ED et al (2018) The Neuronal Gene Arc Encodes a Repurposed Retrotransposon Gag Protein that Mediates Intercellular RNA Transfer Cell 172(2): 275-288.

30. Prasad VR \& BM (2014) New clues to understanding HIV nonprogressors: low cholesterol blocks HIV trans infection. mBio 5(3): 1396-1314.

31. Nguyen DG, Booth A, Gould SJ \& Hildreth JE (2003) Evidence that HIV budding in primary macrophages occurs through the exosome release pathway. J Biol Chem 278(52): 52347-52354.

32. Boker KO et al (2018) The Impact of the CD9 Tetraspanin on Lentivirus Infectivity and Exosome Secretion. Mol Ther 26(2): 634-647.

33. Hakomori SI (2010) Glycosynaptic microdomains controlling tumor cell phenotype through alteration of cell growth, adhesion, and motility. FEBS letters 584(9): 1901-1906.

34. Tint GS et al (1994) Defective cholesterol biosynthesis associated with the Smith-Lemli-Opitz syndrome. N Engl J Med 330(2): 107-113.

35. Sikora DM, Pettit Kekel K, Penfield J, Merkens LS \& Steiner RD (2006) The near universal presence of autism spectrum disorders in children with Smith-Lemli-Opitz syndrome. American journal of medical genetics. Part A 140(14): 1511-1518.

36. Tierney E (2006) Abnormalities of cholesterol metabolism in autism spectrum disorders. American journal of medical genetics. Part B, Neuropsychiatric genetics: the official publication of the International Society of Psychiatric Genetics 141B(6): 666-668.

37. Aneja A, Tierney E (2008) Autism: the role of cholesterol in treatment. International review of psychiatry 20(2): 165-170.

38. Hassan MH, Desoky T, Sakhr HM, Gabra RH \& Bakri AH(2019) Possible Metabolic Alterations among Autistic Male Children: Clinical and Biochemical Approaches. J Mol Neurosci 67(2): 204-216.

39. Benachenhou S, Etcheverry A, Galarneau L, Dube J, Caku A (2019) Implication of hypocholesterolemia in autism spectrum disorder and its associated comorbidities: A retrospective case-control study. Autism Res 12(12): 1860-1869.
40. Grayaa S et al (2018) Plasma oxysterol profiling in children reveals 24-hydroxycholesterol as a potential marker for Autism Spectrum Disorders. Biochimie 153: 80-85.

41. Waage-Baudet $\mathrm{H}$ et al (2003) Abnormal serotonergic development in a mouse model for the Smith-Lemli-Opitz syndrome: implications for autism. Int J Dev Neurosci 21(8): 451-459.

42. Scott MM \& Deneris ES (2005) Making and breaking serotonin neurons and autism. Int J Dev Neurosci 23: 277-285.

43. Incardona JP \& Eaton S (2000) Cholesterol in signal transduction. Current opinion in cell biology 12(2): 193-203.

44. Blassberg R, Macrae JI, Briscoe J \& Jacob J (2016) Reduced cholesterol levels impair Smoothened activation in Smith-Lemli-Opitz syndrome. Hum Mol Genet 25: 693-705.

45. Schengrund CL, Ali Rahmani F\& Ramer JC (2012) Cholesterol, GM1, and autism. Neurochem Res 37(6): 1201-1207.

46. Lekman A, Skjeldal O, Sponheim E \& Svennerholm L (1995) Gangliosides in children with autism. Acta paediatrica 84: 787-790.

47. Nordin V, Lekman A, Johansson M, Fredman P \& Gillberg C (1998) Gangliosides in cerebrospinal fluid in children with autism spectrum disorders. Developmental medicine and child neurology 40(9): 587-594.

48. Mostafa GA \& Al Ayadhi LY (2011) Increased serum levels of antiganglioside M1 auto-antibodies in autistic children: relation to the disease severity. J Neuroinflammation 8: 39.

49. Gonzalez LJ et al (2009) The influence of membrane physical properties on microvesicle release in human erythrocytes. PMC Biophys 2: 7.

50.Wijburg FA, Wegrzyn G, Burton BK \& Tylki Szymanska A (2013) Mucopolysaccharidosis type III (Sanfilippo syndrome) and misdiagnosis of idiopathic developmental delay, attentiodeficit/hyperactivity disorder or autism spectrum disorder. Acta Paediatr 102(5): 462-470.

51. Lamanna WC, Lawrence R, Sarrazin S \& Esko JD (2011) Secondary storage of dermatan sulfate in Sanfilippo disease. J Biol Chem 286(9): 6955-6962.

52. Lamant M \& Apo O (2006) a novel apolipoprotein, is an original glycoprotein up regulated by diabetes in human heart. J Biol Chem 281(47): 36289-36302.

53. Niu M et al (2017) Autism Symptoms in Fragile X Syndrome. J Child Neurol 32(10): 903-909.

54. Berry Kravis E et al (2015) Cholesterol levels in fragile X syndrome. American journal of medical genetics. Part A 167A: 379-384.

55. Caku A (2017) New insights of altered lipid profile in Fragile X Syndrome. PLoS One 12(3): e0174301.

56. Hu VW et al (2009) Gene expression profiling of lymphoblasts from autistic and nonaffected sib pairs: altered pathways in neuronal development and steroid biosynthesis. PLoS One 4(6): e5775.

57. Justice MJ, Buchovecky CM, Kyl SM \& Djukic A (2013) A role for metabolism in Rett syndrome pathogenesis: New clinical findings and potential treatment targets. Rare Dis 1: e27265.

58. Mannini L et al (2015) Mutant cohesin affects RNA polymerase II regulation in Cornelia de Lange syndrome. Sci Rep 5: 16803.

59. Filippakis $\mathrm{H}$ et al (2017) Lysosomal regulation of cholesterol homeostasis in tuberous sclerosis complex is mediated via NPC1 and LDL-R. Oncotarget 8(24): 38099-38112.

60. Stromme P et al (2011) X-linked Angelman-like syndrome caused by Slc9a6 knockout in mice exhibits evidence of endosomal-lysosomal dysfunction. Brain 134(11): 3369-3383.

61. Muller CL, Anacker AMJ, \& Veenstra-VanderWeele J (2016) The serotonin system in autism spectrum disorder: From biomarker to animal models. Neuroscience 321: 24-41. 
62. Chugani DC et al (2002) Role of altered brain serotonin mechanisms in autism. Mol Psychiatry 7 Suppl 2: S16-17.

63. Prasanna X, Jafurulla M, Sengupta D \& Chattopadhyay A (2016) The ganglioside GM1 interacts with the serotonin1A receptor via the sphingolipid binding domain. Biochim Biophys Acta 1858(11): 28182826.

64. Sengupta D \& Chattopadhyay A (2012) Identification of cholesterol binding sites in the serotonin $1 \mathrm{~A}$ receptor. The journal of physical chemistry. B 116: 12991-12996.

65. Paila YD, Chattopadhyay A (2010) Membrane cholesterol in the function and organization of G-protein coupled receptors. Subcellular biochemistry 51: 439-466.

66. Lu AT, Yoon J, Geschwind DH \& Cantor RM (2013) QTL replication and targeted association highlight the nerve growth factor gene for nonverbal communication deficits in autism spectrum disorders. Mol Psychiatry 18(2): 226-235.

67. Fukuda $Y$ et al (2015) Neurotropin promotes NGF signaling through interaction of GM1 ganglioside with Trk neurotrophin receptor in PC12 cells. Brain Res 1596: 13-21.

68. Winden KD, Ebrahimi Fakhari D \& Sahin M (2018) Abnormal mTOR Activation in Autism. Annu Rev Neurosci 41: 1-23.

69. Mathews ES \& Appel B (2016) Cholesterol Biosynthesis Supports Myelin Gene Expression and Axon Ensheathment through Modulation of P13K/ Akt/mTor Signaling. The Journal of neuroscience 36(29): 7628-7639.
70. Kwan V, Unda BK \& Singh KK (2016) Wnt signaling networks in autism spectrum disorder and intellectual disability. Journal of neurodevelopmental disorders 8: 45.

71. Sheng R et al (2014) Cholesterol selectively activates canonical Wnt signaling over non-canonical Wnt signaling. Nature communications 5 : 4393.

72. Coghlan S et al (2012) GABA system dysfunction in autism and related disorders: from synapse to symptoms. Neurosci Biobehav Rev 36(9): 2044-2055.

73. Henin J, Salari R, Murlidaran S \& Brannigan GA (2014) Predicted binding site for cholesterol on the GABAA receptor. Biophysical journal 106(9): 1938-1949.

74. Ngeow J, Sesock K \& Eng C (2017) Clinical Implications for Germline PTEN Spectrum Disorders. Endocrinol Metab Clin North Am 46(2): 503517.

75. Jiang Z, Redfern RE, Isler Y, Ross AH \& Gericke A (2014) Cholesterol stabilizes fluid phosphoinositide domains. Chemistry and physics of lipids 182: 52-61.

76. Cartocci V et al (2018) Altered Brain Cholesterol/Isoprenoid Metabolism in a Rat Model of Autism Spectrum Disorders. Neuroscience 372: 27-37. 